\title{
Control Lyapunov Function Design by Cancelling Input Singularity
}

\author{
Dong Hae Yeom ${ }^{1}$ and Young Hoon $\mathrm{Joo}^{2 *}$ \\ ${ }^{1}$ PostBK21 Team, Kunsan University, Kunsan, Chonbuk, 537-701, Korea \\ ${ }^{2}$ Dept. of Control and Robotics Engineering, Kunsan University, Kunsan, Chonbuk 573-701, Korea
}

\begin{abstract}
If one can find a control Lyapunov function (CLF) for a given nonlinear system, the control input stabilizing the system can be easily obtained. To find a CLF, the time derivative of an energy function should be negative definite. This procedure frequently requires a control input which is a rational function or includes an inverse function. The control input is not defined on the specific state-space where the denominator of the rational function is equal to 0 or the inverse function does not exist. In this region with singularities, the trajectory of the control system cannot be generated, which is one of the most important reasons why it is hard to make the origin of a nonlinear system be globally asymptotically stable. In this paper, we propose a smooth control law ensuring the globally asymptotic stability by means of cancelling the singularity in the control input.
\end{abstract}

Keywords : Control Lyapunov function, input singularity, global stability, smooth control law, cancellation, nonlinear system.

\section{Introduction}

There is general-purpose control scheme for nonlinear systems but it is known that several control schemes are applicable to restricted nonlinear systems which satisfy specific conditions, for example, sliding mode control, feedback linearization, backstepping, etc $[3,4,6]$. In spite of the variety of nonlinear controllers, the stability criterion using an energy function is the most common method. The control Lyapunov function (CLF) approach is an example that such energy function is used actively to design a controller for a nonlinear system [7]. But, this approach has a limitation in ensuring the global stability because a singularity frequently attends the control input. The purpose of this paper is to design a smooth control law which can guarantee the global stability by means of cancelling the singularity of the control input generated in the procedure of CLF design.

One can find a CLF for a given nonlinear system by assigning the control input which makes the time derivative of an energy function selected appropriately be negative definite. In this procedure, the control input involves an inverse function or a rational function. Unfortunately, the control input is not defined on the specific state-space where the inverse function does not exist or the denominator of the rational function is equal to 0 . In this region called the singular manifold, the trajectory of the control system cannot be generated because

Manuscript received Mar. 7, 2012; revised Jun. 11, 2012; accepted Jun. 11, 2012

*Corresponding Author: Young Hoon Joo(yhjoo@kunsan.ac.kr) This work was partially supported by the National Research Foundation of Korea Grant funded by the Korean Government (MEST) (NRF-2009-220-D00034).

(C) The Korean Institute of Intelligent Systems. All rights reserved. the control input diverges. And, in the vicinity of the singular manifold, the control system becomes uncontrollable because the control input is saturated. These divergence and saturation in the control input make it impossible for the trajectory of the control system to across the singular manifold. Thus, the existing CLF approach provides a control law ensuring the local stability around an equilibrium point except the singular manifold. This situation diminishes the virtue of the CLF approach because the local stability also can be achieved by a conventional linear controller based on the linearization of a given nonlinear system around the equilibrium point.

There have been many researches to cope with the singularity problem such as extending the attraction region around the equilibrium point and avoiding the singularity by a discontinuous control input. For example, backstepping is applied to extend a small attraction region by feedback linearization, but the global stability is not guaranteed because the singularity of the resulting control system still remains [6]. Some methods can achieve the global stability by avoiding the singularity, for instance, variable structure control $[8,11]$, sliding mode control [1,3], adaptive control [2], and fuzzy control [5]. The basic idea of these methods is to change the control input or the dynamics of the system for the trajectory of the control system to avoid the singularity when the trajectory approaches the singular manifold. These methods involve a switching action between several control laws or system dynamics, which causes the discontinuity in the control system. The discontinuity may make it difficult to analyze the stability, can cause chattering, and may impose an impulse shock on the system.

In this paper, we propose a method cancelling the singularity generated in the procedure of CLF design. The global stability of the control system is ensured because the proposed control law does not involve the singularity and is well defined on the overall state-space. And, the smooth trajectory of the control 
system is generated because the proposed control scheme does not need a switching action. Finally, some examples are addressed to verify the simplicity and the efficiency of the proposed method compared with feedback linearization, backstepping, and sliding mode control.

\section{Singularity in CLF Approach}

The CLF approach is to assign a control input to make the time derivative of an energy function selected appropriately be negative definite. Consider a nonlinear system with a single input as follows:

$$
\dot{x}=f(x)+g(x) u
$$

Suppose that there is an energy function $V(x)$ which is a continuous and positive definite function. The time derivative of the energy function along the trajectory of the system (1) is

$$
\begin{aligned}
\dot{V} & =\nabla V \dot{x} \\
& =\nabla V[f(x)+g(x) u],
\end{aligned}
$$

where $\nabla V$ denotes the gradient of $V$ with respect to $x$. If the control input $u$ satisfies

$$
u<-\frac{\nabla V f(x)}{\nabla V g(x)}
$$

on the overall state-space except the origin, then $\dot{V}$ is also negative definite except the origin. The existence of this CLF guarantees that the origin of the system is globally asymptotically stable. But, the control input satisfying (2) has a singularity when the denominator is equal to 0 because the control input is given as a rational function. That means the trajectory of the control system cannot be generated on such region called the singular manifold because the control input diverges. Especially, if the singular manifold spans the overall state-space, the trajectory cannot converge to the origin when the initial state is given beyond the singular manifold because the trajectory cannot across that. Thus, one cannot assert the globally asymptotic stability of the origin of the control system. In addition, the control system may become uncontrollable when the trajectory approaches the singular manifold because the control input is saturated before it diverges.

In case of multi-inputs system, the required control inputs are given as not rational functions but inverse functions. The singularity appears when the inverse of a given matrix does not exist, and the saturation may occur when the condition number of a matrix is large $[9,10,12]$.

\section{Cancelling Singularity in CLF Design}

In this paper, we propose a smooth control law which ensures the globally asymptotic stability of the origin of a given nonlinear system by cancelling the input singularity generated in the procedure of CLF design. Consider an affine nonlinear system with a single input $u$ and a constant input matrix $B$ as follows:

$$
\dot{x}=f(x)+B u,
$$

where $x \in \square^{n \times 1}$ is a state variable,$f(x) \in \square^{n \times 1}$ is a drift system which is a smooth vector function, $B \in \square^{n \times 1}$ is a input matrix whose elements are constants, and $u \in \square$ is a control input. Suppose that the drift system $f(x)$ consists of a linear part $A x$ and a nonlinear part $\phi(x)$ which is a real-analytic function. Then, the given nonlinear system can be rewritten as

$$
\dot{x}=A x+\phi(x)+B u .
$$

If there is a smooth control input which makes the time derivative of an energy function be negative definite, the asymptotic stability of the origin of (3) is guaranteed. To extend this argument to the global stability, an additional condition in the energy function is required.

Definition. A function satisfying

$$
V(x) \rightarrow \infty \text { as }\|x\| \rightarrow \infty
$$

is said to be radially unbounded [4].

Lemma. Let the origin be an equilibrium point for (3). If there is a continuously differentiable function $V: \square^{n} \rightarrow \square$ such that

$$
\begin{aligned}
& V(0)=0 \text { and } V(x)>0, \forall x \neq 0, \\
& \dot{V}(x)<0, \forall x \neq 0, \\
& V \text { is radially unbounded. }
\end{aligned}
$$

Then, the origin is globally asymptotically stable [4].

This lemma states the general requirements, that is, the existence of the energy function satisfying (4) guarantees the globally asymptotic stability. But, it is hard to find such energy function for each individual nonlinear system. The following theorem starts from the simplest energy function in the form of a perfect square and concentrates on designing a smooth control input which makes the function be a CLF for a given nonlinear system.

Theorem. For the system (3), if the pair $(A, B)$ is controllable and there is an energy function $V=x^{T} M x$ such that $L_{B} V$ is a factor of $L_{\phi} V$, where $M$ is a symmetric positive definite matrix. Then, there exists a smooth control input which guarantees the globally asymptotic stability of the origin of (3).

Proof. Assign the control input $u$ for the system (3) as

$$
u=-K^{T} x+\rho(x),
$$

where $K \in \square^{n \times 1}$ is a state feedback gain and $\rho(x)$ is a nonlinear state feedback. By this control input the given nonlinear system (3) becomes

$$
\dot{x}=\left(A-B K^{T}\right) x+\phi(x)+B \rho(x) .
$$

Meanwhile, the time derivative of an energy function $V$ along the trajectory of the system is 


$$
\begin{aligned}
\dot{V} & =\nabla V \dot{x} \\
& =\nabla V\left[\left(A-B K^{T}\right) x+\phi(x)+B \rho(x)\right] \\
& =L_{\Gamma x} V+L_{\phi(x)} V+L_{B} V \rho(x),
\end{aligned}
$$

where $\nabla V \in \square^{1 \times n}$ is the gradient of $V$ with respect to $x$, $L_{h} V$ is the Lie derivative of $V$ along $h$, and $\Gamma=A-B K^{T}$. There exists a state feedback gain $K$ which allows $\Gamma$ to be a Hurwitz matrix because the pair $(A, B)$ is controllable. Let $L_{\phi} V=a(x), L_{B} V=b(x)$, and $L_{\Gamma x} V=c(x)$, then

$$
\dot{V}=c(x)+a(x)+b(x) \rho(x) .
$$

Using an energy function $V=x^{T} M x$ which satisfies the first condition of (4), the first term of the right hand side of (6) is

$$
c(x)=x^{T}\left(M \Gamma+\Gamma^{T} M\right) x .
$$

Because $\Gamma$ is a Hurwitz matrix there exist a symmetric positive definite matrix $N$ such that

$$
M \Gamma+\Gamma^{T} M=-N,
$$

which yields $c(x)<0$ for all $x$ except the origin.

If the nonlinear state feedback of (5) and (6) is assigned as

$$
\rho(x)=-\frac{a(x)}{b(x)},
$$

then the time derivative of the energy function (6) is less than 0 for all $x$ except the origin and the singular manifold $S=\{x \mid b(x)=0\}$. By the assumption, $b(x)$ is a factor of $a(x)$, the denominator of (7) is cancelled out. This leads to the result that the control input (5) is a smooth function and well defined on the overall state-space, which satisfies the second condition of (4) and the continuous differentiability of $V(x)$.

The third condition of (4) is confirmed by Rayleigh inequality as follows:

$$
\lambda_{\min }(M)\|x\|^{2} \leq V \leq \lambda_{\max }(M)\|x\|^{2},
$$

where $\lambda_{\min }$ and $\lambda_{\max }$ denote the minimum and the maximum eigenvalue, which are real numbers because $M$ is a symmetric matrix. As $\|x\|$ diverges to infinity, the lower bound diverges and so does the energy function. Therefore, the smooth control input (5) allows the energy function $V$ to be a CLF for the given system and guarantees the globally asymptotic stability of the origin.

Remark. This theorem is a special case of Artstein's theorem which states that the existence of a smooth CLF implies smooth stabilizability [7]. The author therein proposed a feedback control law

$$
u=-\frac{L_{f} V+\sqrt{\left(L_{f} V\right)^{2}+\left(L_{B} V\right)^{4}}}{L_{B} V}
$$

which provides a proof of Artstein's theorem. The singularity in this control law does not appear as long as $V$ satisfies the small control property around the origin, that is, the denominator $L_{B} V$ is sufficiently small when $x$ is small. This work addressed a conceptual approach based on the existence of a CLF but did not show how to design such a CLF.

\section{Examples}

In general, it is not easy to find an appropriate CLF for a given nonlinear system. In this paper, we concentrate on designing a control input which makes the simplest energy function in the form of a perfect square be a CLF. The following examples show that the proposed method provides a smooth control law guaranteeing the globally asymptotic stability unlike the existing methods which have a limitation in ensuring the global stability because of the singularity in the control input or the smooth trajectory because of the discontinuity in the control input.

\subsection{Local Stability by Feedback Linearization}

This example is the case that the region of attraction is restricted because of the singularity in the control input designed by using feedback linearization.

$$
\begin{aligned}
& \dot{x}_{1}=-x_{1}+x_{2}-x_{3} \\
& \dot{x}_{2}=-x_{1} x_{3}-x_{2}+u . \\
& \dot{x}_{3}=-x_{1}+u
\end{aligned}
$$

The resulting control input by feedback linearization is given as

$$
u=\frac{1}{x_{1}+1}\left(v-3 x_{1}+4 x_{2}-2 x_{3}-x_{2} x_{3}+3 x_{1} x_{3}+x_{1}^{2}+x_{3}^{2}\right) .
$$

By this control input, the nonlinear system (8) becomes a linear system with a new control input $v$ in (9). The asymptotic stability of the origin of the system can be achieved by an arbitrary state feedback which stabilizes the linearized system [4]. However, because the control input (9) has the singular manifold $S=\left\{x \mid x_{1}+1=0\right\}$, the control input is not defined on this manifold. And the manifold spans the overall statespace. Thus, the global stability of the control system is not ensured. For example, the trajectory starting from an initial state $x_{0}=\left[\begin{array}{lll}-2 & 0 & 0\end{array}\right]^{T}$ can not converge to the origin because it can not across the singular manifold.

- To apply the proposed method, rewriting the system (8) in the form of (3) yields

$$
\begin{aligned}
\dot{x} & =A x+B u+\phi(x) \\
& =\left[\begin{array}{ccc}
-1 & 1 & -1 \\
0 & -1 & 0 \\
-1 & 0 & 0
\end{array}\right] x+\left[\begin{array}{l}
0 \\
1 \\
1
\end{array}\right] u+\left[\begin{array}{c}
0 \\
-x_{1} x_{3} \\
0
\end{array}\right] .
\end{aligned}
$$

The pair $(A, B)$ of the system is controllable because the controllability matrix has full rank. Let an energy function be $V=x^{T} M x$, where $M$ is an arbitrary symmetric positive definite matrix as follows: 


$$
M=\left[\begin{array}{lll}
m_{11} & m_{12} & m_{13} \\
m_{12} & m_{22} & m_{23} \\
m_{13} & m_{23} & m_{33}
\end{array}\right] .
$$

And, calculate $L_{\phi} V$ and $L_{B} V$.

$$
\begin{aligned}
& L_{\phi} V=-2 x_{1} x_{3}\left(m_{12} x_{1}+m_{22} x_{2}+m_{23} x_{3}\right), \\
& L_{B} V=2\left[\left(m_{12}+m_{13}\right) x_{1}+\left(m_{22}+m_{23}\right) x_{2}+\left(m_{23}+m_{33}\right) x_{3}\right] .
\end{aligned}
$$

Assign the elements of $M$ to make $L_{B} V$ be $x_{3}$ which is a factor of $L_{\phi} V$. For example, $m_{12}=-m_{13}, m_{22}=-m_{23}$, and the other elements are chosen arbitrarily for $M$ to be positive definite as follows:

$$
M=\left[\begin{array}{ccc}
2 & 1 & -1 \\
1 & 1 & -1 \\
-1 & -1 & 3
\end{array}\right]>0 .
$$

This symmetric positive definite matrix allows

$$
\begin{aligned}
& L_{\phi} V=-2 x_{1} x_{3}\left(x_{1}+x_{2}-x_{3}\right) \\
& L_{B} V=4 x_{3}
\end{aligned}
$$

to satisfy the assumption of Theorem in Section 3 and the energy function $V$ to be a CLF for the given system. The resulting control input by (5), (7), and (10) is

$$
u=-K^{T} x+\frac{1}{2} x_{1}\left(x_{1}+x_{2}-x_{3}\right),
$$

where $K$ is a state feedback gain for $A-B K^{T}$ to be stable. Unlike the control input (9) by feedback linearization, there is no singularity in the proposed control input (11). Thus, the globally asymptotic stability of the origin of the system (8) is guaranteed by the proposed smooth control input.

\subsection{Extending Attraction Region by Backstepping}

This example is the case that the region of attraction is extended by using backstepping, however, the singularity still remains.

$$
\begin{aligned}
& \dot{x}_{1}=x_{2} \\
& \dot{x}_{2}=x_{1}+\left(1+x_{1}+x_{2}\right) x_{3} . \\
& \dot{x}_{3}=u
\end{aligned}
$$

The resulting control input by backstepping is given as

$$
\begin{aligned}
z_{1}= & x_{1} \\
z_{2}= & x_{2}+\varepsilon_{1} x_{1} \\
z_{3}= & x_{3}+\frac{1}{1+x_{1}+x_{2}}\left(\varepsilon_{2} z_{2}+\varepsilon_{1} x_{2}+2 x_{1}\right) \\
u= & -\varepsilon_{3} z_{3}-\frac{\varepsilon_{1}+\varepsilon_{2}}{\left(1+x_{1}+x_{2}\right)^{2}}\left[x_{1}+x_{2}+\left(1+x_{1}+x_{2}\right) x_{3}\right] \\
& -\left(1+x_{1}+x_{2}\right) z_{2},
\end{aligned}
$$

where $\varepsilon_{1}, \varepsilon_{2}, \varepsilon_{3}$ are design parameters [6]. The control input $u$ in (13) includes a rational function, thus $u$ is not defined on the singular manifold $S=\left\{x \mid x_{1}+x_{2}+1=0\right\}$, which obstructs the global stability of the control system.

To apply the proposed method, rewriting the system (12) in the form of (3) yields

$$
\begin{aligned}
\dot{x} & =A x+B u+\phi(x) \\
& =\left[\begin{array}{ccc}
-1 & 1 & -1 \\
0 & -1 & 0 \\
-1 & 0 & 0
\end{array}\right] x+\left[\begin{array}{l}
0 \\
1 \\
1
\end{array}\right] u+\left[\begin{array}{c}
0 \\
-x_{1} x_{3} \\
0
\end{array}\right]
\end{aligned}
$$

The pair $(A, B)$ of the system is controllable because the controllability matrix has full rank. Let an energy function be $V=x^{T} M x$, where $M$ is an arbitrary symmetric positive definite matrix. And calculate $L_{\phi} V$ and $L_{B} V$.

$$
\begin{aligned}
& L_{\phi} V=2 x_{3}\left(x_{1}+x_{2}\right)\left(m_{12} x_{1}+m_{22} x_{2}+m_{23} x_{3}\right) \\
& L_{B} V=2\left(m_{13} x_{1}+m_{23} x_{2}+m_{33} x_{3}\right)
\end{aligned}
$$

Assign the elements of $M$ to make $L_{B} V$ be $x_{3}$ which is a factor of $L_{\phi} V$. For example, put $m_{13}=m_{23}=0$ and the other elements are chosen arbitrarily for $M$ to be positive definite as follows:

$$
M=\left[\begin{array}{lll}
2 & 1 & 0 \\
1 & 1 & 0 \\
0 & 0 & 1
\end{array}\right]>0
$$

This symmetric positive definite matrix allows

$$
\begin{aligned}
& L_{\phi} V=2\left(x_{1}+x_{2}\right)^{2} x_{3} \\
& L_{B} V=2 x_{3}
\end{aligned}
$$

to satisfy the assumption of Theorem in Section 3 and the energy function $V$ to be a CLF for the given system. The resulting control input by (5), (7), and (14) is

$$
u=-K^{T} x-\left(x_{1}+x_{2}\right)^{2},
$$

where $K$ is a state feedback gain for $A-B K^{T}$ to be stable. Unlike the control input in (13) by backstepping, there is no singularity in the proposed control input (15). Thus, the globally asymptotic stability of the origin of the system (12) is guaranteed by the proposed smooth control input.

\subsection{Global Stability by Sliding Mode Control}

This example is the case that the globally asymptotic stability is achieved by sliding mode control, however, the trajectory of the control system is not smooth because of the discontinuity in the control input.

$$
\begin{aligned}
& \dot{x}_{1}=x_{3}^{2}-x_{2} \\
& \dot{x}_{2}=x_{3} \\
& \dot{x}_{3}=u
\end{aligned} .
$$


The resulting control input by sliding mode control is given as

$$
\begin{aligned}
& \alpha(x)=x_{3}^{2}-x_{2}-x_{3} \\
& \beta(x)=2 x_{3} \\
& u_{s m}=-\frac{(\alpha+k) \operatorname{sign}\left(x_{1}+x_{3}^{2}-x_{2}\right)}{\beta} \\
& u=\min \left\{u_{s m},-l \operatorname{sign}\left[\left(x_{1}+x_{3}^{2}-x_{2}\right) \beta\right]\right\},
\end{aligned}
$$

where $k$ and $l$ are design parameters [3]. The discontinuity in $u$ of (17) makes it difficult to analyze the stability, can causes chattering, and may impose an impulse shock on the system. From a practical point of view, the chattering and the impulse shock are the main sources of noise.

Rewriting the system (16) in the form of (3) yields

$$
\begin{aligned}
\dot{x} & =A x+B u+\phi(x) \\
& =\left[\begin{array}{ccc}
0 & -1 & 0 \\
0 & 0 & 1 \\
0 & 0 & 0
\end{array}\right] x+\left[\begin{array}{l}
0 \\
0 \\
1
\end{array}\right] u+\left[\begin{array}{c}
x_{3}^{2} \\
0 \\
0
\end{array}\right] .
\end{aligned}
$$

The pair $(A, B)$ of the system is controllable because the controllability matrix has full rank. Let an energy function be $V=x^{T} M x$, where $M$ is an arbitrary symmetric positive definite matrix. And calculate $L_{\phi} V$ and $L_{B} V$.

$$
\begin{aligned}
& L_{\phi} V=2\left(m_{11} x_{1}+m_{12} x_{2}+m_{13} x_{3}\right) x_{3}^{2} \\
& L_{B} V=2\left(m_{13} x_{1}+m_{23} x_{2}+m_{33} x_{3}\right)
\end{aligned}
$$

Assign the elements of $M$ to make $L_{B} V$ be $x_{3}$ which is a factor of $L_{\phi} V$. By the same manner in the previous section, put $m_{13}=m_{23}=0$ and the other elements are chosen arbitrarily for $M$ to be positive definite as follows:

$$
M=\left[\begin{array}{lll}
2 & 1 & 0 \\
1 & 1 & 0 \\
0 & 0 & 1
\end{array}\right]>0
$$

This symmetric positive definite matrix allows

$$
\begin{aligned}
& L_{\phi} V=2\left(2 x_{1}+x_{2}\right) x_{3}^{2} \\
& L_{B} V=2 x_{3}
\end{aligned}
$$

to satisfy the assumption of Theorem in Section 3 and the energy function $V$ to be a CLF for the given system. The resulting control input by (5), (7), and (18) is

$$
u=-K^{T} x-\left(2 x_{1}+x_{2}\right) x_{3},
$$

where $K$ is a state feedback gain for $A-B K^{T}$ to be stable. Unlike the control input in (17) by sliding mode control, there is no discontinuity in the proposed control input (19). Thus, the proposed method provides a smooth control input which guarantees the globally asymptotic stability of the origin of the system (16).

\section{Conclusions}

In this paper, we proposed a control method by cancelling the input singularity generated in the procedure of CLF design. The proposed method guaranteed the globally asymptotic stability and provided a smooth control law unlike the existing methods such as feedback linearization, backstepping, and sliding mode control. The examples showed the simplicity and the efficiency of our approach. However, the singularity was represented as a linear combination because of the assumption that the input matrix is a constant and that the energy function is a perfect square form. This caused fewer choices in cancellation terms and restrictions on applicable systems. Further research is required to improve the applicability of the method by considering more various energy functions

\section{References}

[1] B. Castillo-Toledo and A. L. Cuevas, "Tracking through Singularities Using a Robust Differentiator," 6th Int. Conf. Electric. Eng., Comp. Sci., Autom. Control, pp. 1-5, Jan. 2009.

[2] D. Colon and F. M. Pait, "Geometry of Adaptive Control : Optimization and Geodesics," Int. J. Adapt. Control, Signal Process., vol. 18, no. 4, pp. 381-392, Apr. 2004.

[3] R. M. Hirschorn, "Output Tracking through Singularities," Proc. 41st IEEE CDC, pp. 3843-3848, Dec. 2002.

[4] Khalil, Nonlinear Systems, 3rd Ed., Prentice Hall, New Jersey, 2002.

[5] X. Z. Lai, S. X. Yang, J. H. She, and M. Wu, "Singularity Avoidance for Acrobots Based on Fuzzy-control Strategy," Robot., Autonom. Syst., vol. 57, no. 2, pp. 202-211, Feb. 2009.

[6] Z. H. Li and M. Krstic, "Maximizing Regions of Attraction via Backstepping and CLFs with Singularities," Syst., Control Lett., vol. 30, no. 4, pp. 195-207, May 1997.

[7] D. E. Sontag, "A Universal Construction of Artstein Theorem on Nonlinear Stabilization," Syst., Control Lett., vol. 13 , no. 2, pp. 117-123, Aug. 1989.

[8] C. J. Tomlin and S. S. Sastry, "Switching through Singularities," Syst., Control Lett., vol. 35, no. 3, pp. 145154, Oct. 1998.

[9] H. Wei and S. I. Amari, "Dynamics of Learning Near Singularities in Radial Basis Function Networks," Neur. Net., vol. 21, no. 7, pp. 989-1005, Sep. 2008.

[10] L. Xiaoping and S. Celikovsky, "Feedback Control of Affine Nonlinear Singular Control Systems," Int. J. Control, vol. 68, no. 4, pp. 753-774, 1997.

[11] J. X. Xu, T. H. Lee, and M. Wang, "Self-Tuning Type Variable Structure Control Method for a Class of Nonlinear Systems," Int. J. Robust, Nonlinear Control, vol. 8, no. 13, pp. 1133-1153, Dec. 1998.

[12] J. X. Xu and R. Yan, "Fixed Point Theorem-Based Iterative Learning Control for LTV Systems with Input 
Singularity," IEEE Trans. Autom. Control, vol. 48, no. 3, pp. 487-492, Mar. 2003.

[13] G. B. Koo, J. B. Park, and Y. H. Joo, "Observer-based Sampled-data controller of linear system for the wave energy converter," Int. Journal of Fuzzy Logic and Intelligent Systems, vol.11, no. 4, pp. 275-279, Dec. 2011.

\section{Dong Hae Yeom}

Research Professor of the Kunsan National University
Research Area: nonlinear systems, and switching and hybrid control, intelligent control and modeling, etc.

E-mail : flyht@korea.com

\section{Young Hoon Joo}

Professor of the Kunsan National University

Research Area: Intelligent robot system control, intelligent control and modeling, nonlinear system control, intelligent emotion recognition, and WEB-based virtual laboratory, etc.

E-mail : yhjoo@kunsan.ac.kr 